\title{
Erratum to: The impact of decentralization policies: the environmental performance applied to municipalities of the Amazon
}

\section{Errata para: $O$ impacto de políticas descentralizadas: o desempenho ambiental aplicado a municípios da Amazônia}

Benedito Evandro Barros da SILVA ${ }^{1,2^{*}}$, Claudia AZEVEDO-RAMOS ${ }^{3}$, Hilder André Bezerra FARIAS ${ }^{4}$

${ }^{1}$ Programa de Pós-Graduação em Desenvolvimento Sustentável dos Trópicos Úmidos, Universidade Federal do Pará (UFPA), Belém, PA, Brasil.

${ }^{2}$ Secretaria de Estado de Meio Ambiente do Pará (SEMAS/PA), Belém, PA, Brasil.

${ }^{3}$ Núcleo de Altos Estudos da Amazônia (NAEA), Universidade Federal do Pará (UFPA), Belém, PA, Brasil.

${ }^{4}$ Departamento de Economia, Universidade Federal do Pará (UFPA), Belém, PA, Brasil.

*E-mail of contact: evandroourem @ yahoo.com.br

Article received on September 22, 2018, final version accepted on May 23, 2019, published on August 2019.

The original article can be found online at: http://dx.doi.org/10.5380/dma.v51i0.61705

References have been corrected, as follows:

Andersen, L. E.; Reis, E. J. Deforestation, development, and government policy in the Brazilian Amazon: an econometric analysis. Discussion Paper 69. Institute for Applied Economic Research - IPEA, 2015. Available at: http://repositorio.ipea.gov.br/bitstream/11058/4832/1/DiscussionPaper_69.pdf. Accessed in: nov. 2017.

Andersson, K.; Gibson, C.; Lehoucq., F. Municipal politics and forest governance: comparative analysis of decentralization in Bolivia and Guatemala. World Development, 34(3), 576-95, 2006. doi: 10.1016/j.worlddev.2005.08.009 
Andersson, K.; Laerhoven, F. V. From local strongman to facilitator: institutional incentives for participatory municipal governance in Latin America. Comparative Political Studies, 40(7), 1085-1111, 2007. doi: 10.1177/0010414006288977

Azevedo, A. A.; Rajão, R. L.; Costa, M.; Stabeli, M. C. C.; Macedo, M. N.; Dos Reis, T. N. P.; Alencar A.; Soares-Filho, B.; Pacheco, R. Limits of Brazil's Forest Code as a means to end illegal deforestation. PNAS, 114(29), 7653-7658. 2017. doi: 10.1073/pnas.1604768114

BACEN - Banco Central do Brasil. Crédito Rural. Ano base 2009 e 2013. 2017. Available at: http://www.bcb.gov.br/pt-br/\#!/n/CREDRURAL. Accessed in: May. 2017.

Barona, E.; Ramankutty, N.; Hyman, G.; Coomes, O. T. The role of pasture and soybean in deforestation of the Brazilian Amazon. Environmental Research Letters, 5, 024002 , 2010. doi: 10.1088/1748-9326/5/2/024002/meta

Bomfim, S. L. Índice de clima econômico para concessões florestais. $\mathrm{PhD}$ thesis. Universidade de Brasília (UNB), 2014. Available at: http://repositorio.unb.br/handle/10482/17230. Accessed in: Aug. 2017.

Celentano, D.; Veríssimo, A. O Avanço da Fronteira na Amazônia: do boom ao colapso. O Estado da Amazônia - Indicadores, 48. Belém: Imazon, 2007.

Coleman, E. A.; Fleischman, F. D. Comparing Forest Decentralization and Local Institutional Change in Bolivia, Kenya, Mexico, and Uganda. World Development, 40(4), 836-849, 2012. doi: 10.1016/j.worlddev.2011.09.008

Costello, A. B.; Osborne J. W. Best practices in exploratory factor analysis: Four recommendations for getting the most from your analysis. Practical assessment, research \& evaluation. 2005. Available at: http://pareonline.net/getvn.asp? $\mathrm{v}=10 \& \mathrm{n}=7$

De Lima, I. B.; Buszynski, L. Local environmental governance, public policies and deforestation in Amazonia. Management of Environmental Quality: An International Journal, 22(3), 292-316, 2011. doi: 10.1108/14777831111122888

Falleti, T. Decentralization and subnational politics in Latin America. Cambridge University Press, Cambridge, 2010.

Fapespa - Fundação Amazônia de Amparo a Estudos e Pesquisas. (2014) Estatística Municipal do Pará 2014. Available at: <http://www.fapespa.pa.gov.br/produto>. Accessed in: May. 2017.

Fapespa - Fundação Amazônia de Amparo a Estudos e Pesquisas. Anuário Estatístico do Pará 2015, 2015. Available at: <http://www.fapespa.pa.gov.br/anuario_estatistico> Accessed in: May. 2017.

FIRJAN - Índice FIRJAN de Desenvolvimento Municipal. Federação das Indústrias do Estado do Rio de Janeiro. 2015. Available at: <http://www.firjan.com.br/ifdm/downloads/>. Accessed in: Oct. 2017.

Forgy, E.W. Cluster analysis of multivariate data: efficiency versus interpretability of classifications. Biometrics, 21,768-769, 1965. 
Galbadón, A. J. La descentralización de la gestón ambiental en América Latina. Reforma y Democracia, 45,1-77, 2009.

Garcia, E.; Ramos Filho.; F. S. V; Mallmann, G. M; Fonseca, F. Costs, benefits and challenges of sustainable livestock intensification in a major deforestation frontier in the Brazilian Amazon. Sustainability, 9(1), 158, 2017. doi: 10.3390/su9010158

Gibson, C.; Lehoucq, F. The local politics of decentralized environmental policy in Guatemala. Journal of Environment and Development, 12(1), 28-49, 2003. doi: $10.1177 / 1070496502250437$

Guedes, G.; Costa, S.; Brondízio, E. Revisiting the hierarchy of urban areas in the Brazilian Amazon: a multilevel approach. Population and Environment, 30(4-5), 159192, 2009.

Hair, J.; Black, W.; Babin, B.; Anderson, R.; Tatham, R. Multivariate data analysis (6th ed.). Pearson Prentice Hall. Uppersaddle River, N.J, 2006.

Hartigan, J. A. Clustering algorithms. John Willey and Sons. New York, NY, 1975.

Hecht, S. B. The Logic of Livestock and Deforestation in Amazonia. BioScience, 43(10), 687-695, 1993. doi: 10.2307/1312340

IBGE - Instituto Brasileiro de Geografia e Estatística. Indicadores de desenvolvimento sustentável. $2015 . \quad$ Available at: <https://ww2.ibge.gov.br/home/geociencias/recursosnaturais/ids/default_2015.shtm> Accessed in: Jan. 2017.

IBGE - Instituto Brasileiro de Geografia e Estatística. SIDRA. 2017a. Available at: <http://www.sidra.ibge.gov.br>. Accessed in: Sept. 2017.

IBGE - Instituto Brasileiro de Geografia e Estatística. Pesquisa de Informações Básicas Municipais. 2017b. Available at: <https://ww2.ibge.gov.br/home/estatistica/economia/perfilmunic/defaulttab1_perfil.sht $\mathrm{m}>$ Accessed in: Feb. 2017.

IBGE - Instituto Brasileiro de Geografia e Estatística. Taxa de crescimento populacional. 2017c. Available at: <ftp://ftp.ibge.gov.br> Accessed in: Feb. 2017.

IBGE - Instituto Brasileiro de Geografia e Estatística. IBGE Cidades, 2017d. Available at: <https://cidades.ibge.gov.br/> Accessed in: Dec. 2017.

INPE - Instituto Nacional de Pesquisas Espaciais. DEGRAD - Mapeamento da Degradação Florestal na Amazônia Brasileira. 2017b. Available at: <http://www.obt.inpe.br/degrad//>. Accessed in: August 2017.

INPE - Instituto Nacional de Pesquisas Espaciais. PRODES - Sistema de Monitoramento do Desmatamento na Amazônia Legal. 2017a. Available at: <http://www.dpi.inpe.br/prodesdigital/prodes.php> Accessed in: Aug. 2017. 
INPE - Instituto Nacional de Pesquisas Espaciais. Projeto Terraclass. 2017c. Available at: <http://www.inpe.br/cra/projetos_pesquisas/dados_terraclass.php>. Accessed in: Aug. 2017.

IPS Amazônia - Índice de Progresso Social na Amazônia Brasileira. 2014. Belém: Imazon e Social Progress Imperative. Available at: $<\mathrm{http}: / / \mathrm{www}$.ipsamazonia.org.br/\#aspects $\% 5 \mathrm{~B} \% 5 \mathrm{D}=1 \&$ aspects $\% 5 \mathrm{~B} \% 5 \mathrm{D}=2 \&$ aspects $\%$ $5 \mathrm{~B} \% 5 \mathrm{D}=7 \&$ aspects $\% 5 \mathrm{~B} \% 5 \mathrm{D}=12 \&$ map-view=city\&map-type=performance \&activecat $=1 \&$ page $=1 \&$ tab $=$ map $>$. Accessed in: Jun. 2018.

Johnson, R. A.; Wichern, D. W. Applied multivariate statistical analysis. Prentice-Hall. Upper Saddle River, NJ, 2015.

Kaimowitz, D.; Mertens, B.; Wunder, S.; Pacheco, P. Hamburger connection fuels Amazon destruction. Center for International Forestry Research, 2004. Available at: $\langle$ http://www.cifor.cgiar.org/publications/pdf_files/media/Amazon.pdf $>$. Accessed in: Sept. 2017.

Larsen, P. B. Municipal environmental governance in the Peruvian Amazon: A case study in local matters of (in) significance. Management of Environmental Quality: An International Journal, 22(3),374-385, 2011. doi: 10.1108/14777831111122932

Lloyd, S. P. Least squares quantization in PCM. IEEE Transactions on Information Theory. 1982. Available at: <http://ieeexplore.ieee.org/document/1056489/> Accessed in: Nov. 2017.

Meijering, E. A chronology of interpolation: from ancient astronomy to modern signal and image processing. Proceedings of the IEEE, 90(3), 319-342, 2002.

MMA - Ministério do Meio Ambiente. Lista de Municípios Prioritários da Amazônia. 2013 Available at: <http://www.mma.gov.br/images/arquivos/florestas/controle_e_prevencao/2014/lista_\% 20municipios_\%20prioritarios_07.08.pdf>. Accessed in: Sept. 2017.

Mori, K.; Christodoulou, A. Review of sustainability indices and indicators: Towards a new city sustainability index (CSI). Environmental Impact Assessment Review, 32, 94106, 2012. doi: 10.1016/j.eiar.2012.06.001

Moutinho, P.; Guerra, R.; Azevedo-Ramos, C. Achieving zero deforestation in the Brazilian Amazon: what is missing? Elementa: Science of the Anthropocene, 2016. doi: 10.12952/journal.elementa.000125

MS - Ministério da Saúde. DataSus - Sistema de Informações sobre Mortalidade, 2015. Available at: <http://tabnet.datasus.gov.br/cgi/deftohtm.exe?sim/cnv/pevita10PA.def> Accessed in: Febr. 2017.

Nepstad, D.; McGrath, D.; Stickler, C.; Alencar, A.; Azevedo, A.; Swette, B.; Bezerra, T.; DiGiano, M.; Shimada, J.; Motta, R. S. da.; Armijo, E.; Castello, L.; Brando, P.; Hansen, M. C.; McGrath-Horn, M.; Carvalho, O.; Hess, L. Slowing Amazon deforestation through public policy and interventions in beef and soy supply chains. Science, 344(6188), 118-123, 2014. doi: 10.1126/science.1248525 
Nepstad, D.; Veríssimo, A.; Alencar, A.; Nobre, C.; Lima, E.; Lefebvre, P.; Schlesinger, P.; Potter, C.; Moutinho, P.; Mendoza, E.; Cochrane, M.; Brooks, V. Large-scale impoverishment of Amazonian forests by logging and fire. Nature, 398, 505-508, 1999.

Nicole, A. G.; Tronchin D. M. R. Indicadores para avaliação do acesso vascular de usuários em hemodiálise. Revista da Escola de Enfermagem da USP, 45(1), 206-214, 2011. doi: 10.1590/S0080-62342011000100029

Persha, L.; Andersson, K. Elite capture risk and mitigation in decentralized forest governance regimes. Global Environmental Change, 24, 265-276, 2014.

PMV - Programa Municípios Verdes. Atividades e Resultados 2014/2015. Governo do Estado do Pará, $2016 . \quad$ Available at: <http://www.municipiosverdes.pa.gov.br/relatorios/seleciona_tipo> Accessed in: Jun. 2017.

Schmitt, J.; Scardua, F. P. The environmental decentralization and the enforcement of Amazon deforestation. Revista de Administração Pública, 49,1121-1142, 2015. doi: 10.1590/0034-7612131456

SEMAS - Secretaria de Estado de Meio Ambiente e Sustentabilidade. Cadastro Ambiental Rural (2009 and 2015, Pará. Brazil). 2017b. Available at: <http://car.semas.pa.gov.br/\#/>. Accessed in: Nov. 2017.

SEMAS - Secretaria de Estado de Meio Ambiente e Sustentabilidade. Relação de Municípios que possuem capacidade para exercer a gestão ambiental de forma plena elou parcial. Pará. Brazil. (2017a). Available at: <https://www.semas.pa.gov.br/wpcontent/uploads/2017/12/municipios-gestao-ambiental-21-12-17.pdf > Accessed in: Aug. 2017.

Silva, B. E. B. da.; Azevedo-Ramos, C. Evolução da Descentralização da Gestão Ambiental Municipal no Estado do Pará. In: Pezzuti J, Azevedo-Ramos C. (Eds.). Desafios Amazônicos. Série Desenvolvimento e Sustentabilidade. NAEA editora. Belém, Pará. Brazil. Pp 261-296, 2016.

Singh, R. K.; Murty, H. R.; Gupta, S. K.; Dikshit, A. K. An overview of sustainability assessment methodologies. Ecological Indicators, 9(2), 189-212, 2009.

Thompson, B. Exploratory and confirmatory factor analysis: Understanding concepts and applications. American Psychological Association, Washington, DC, 2004.

Viana, C.; Coudel, E.; Barlow, J.; Ferreira, J. N.; Gardner, T. A.; Parry, L. How does hybrid governance emerge? Role of the elite in building a green municipality in the Eastern Brazilian Amazon. Environmental Policy and Governance, 26(5), 337-350, 2016.

Wood, C. H.; Perz, S. G. Population and land-use changes in the Brazilian Amazon. In: Ramphal, S. D.; Sinding S. W. (Eds.). Population growth and environmental issues. Praeger. Westport, Connecticut. Pp. 95-108, 1996.

Zarin, D. J.; Harris, N. L.; Baccini, A.; Aksenov, D.; Hansen, M. C.; Azevedo-Ramos, C.; Azevedo, T.; Margono, B. A.; Alencar, A. C.; Gabris, C.; Allegretti, A.; Potapov, P.; 
Farina, M.; Walker, W.S.; Shevade, V.S.; Loboda, T.V.; Turubanova, S.; Tyukavina, A. Can Carbon emissions from tropical deforestation drop by $50 \%$ in 5 years? Global Change Biology, 22(4), 1336-1347, 2016. doi: 10.1111/gcb.13153

Published on: September, 22, 2020.

DOI: $10.5380 /$ dma.v51i0.76698 